\title{
THE ISOTOPIC COMPOSITION OF COSMIC RAY NUCLEI BEYOND THE IRON PEAK
}

\author{
R. A. Mewaldt and E. C. Stone \\ California Institute of Technology, Pasadena, CA 91125 \\ W. R. Binns and J. Klarmann \\ Washington University, St. Louis, MO 63130
}

\begin{abstract}
Isotope measurements of cosmic ray nuclei beyond the Fe peak are considered, using the charge region from $\mathrm{Z}=\mathbf{2 9}$ to $\mathrm{Z} \sim \mathbf{4 0}$ as an example. Such studies can address a number of important questions that bear on cosmic ray origin, acceleration, and propagation. One possible approach for measuring isotopes with $\mathrm{Z} \geq 30$ is based on large-area arrays of silicon solid state detectors combined with scintillating optical fiber trajectory detectors.
\end{abstract}

\section{INTRODUCTION}

Studies of the isotopic composition of cosmic rays heavier than $\mathrm{Fe}(\mathrm{Z}=26)$, including $\mathrm{Co}, \mathrm{Ni}$, and $\mathrm{Cu}(\mathrm{Z}=27$ to 29$)$ and the ultraheavy (UH) nuclei $(\mathrm{Z} \geq 30)$ although complicated by the relative rarity of these nuclei, can address a number of important objectives. Over the next decade we can expect missions such as the Advanced Composition Explorer ${ }^{1}$ (ACE) to provide precision measurements of cosmic ray isotopes from $\mathrm{H}$ to nickel ( $\mathrm{Z}=1$ to 28 ), and the HNC experiment ${ }^{2}$ on the Space Station to provide greatly improved information on the composition of UH elements with $\mathrm{Z} \geq 60$. We consider here, as an example, the significance of isotope measurements of nuclei with $\mathrm{Z}=29$ to $\mathrm{Z} \sim \mathbf{4 0}$, the next most accessible region for further isotope studies. Similar considerations apply to heavier nuclei.

\section{COSMIC RAY NUCIEI HEAVIER THAN IRON}

One basic question that can be addressed by UH isotope studies is whether cosmic ray nuclei in the upper $2 / 3$ of the periodic table have a common origin and history with that of lighter nuclei. The nucleosynthesis of nuclei with $\mathrm{Z}>30$ is dominated by the rapid and slow ( $\mathrm{r}$ and $\mathrm{s}$ ) neutron capture processes, but also includes contributions from equilibrium burning (e-process) and the proton capture (p) process. HEAO-3 and Ariel-VI elemental composition studies suggest that cosmic ray nuclei with $32 \leq \mathrm{Z} \leq 60$ contain a somewhat higher proportion of r-process material than does the solar system, while the r-process contribution to nuclei with $Z>60$ apparently substantially exceeds that in the solar system ${ }^{3}$.

Such questions are much more easily addressed with isotope studies, since these nucleosynthesis processes do not in general produce the same isotopes of a given element. Figure 1 shows "solar system" abundances" of isotopes with $30 \leq \mathrm{Z} \leq 40$, including an indication of the dominant nucleosynthesis process 
contributing to each isotope. Note that while the production of some elements such as $\mathrm{Ga}$, Se, and $\mathrm{Sr}$ is dominated by a single process, others, such as $\mathrm{Zn}, \mathrm{Ge}, \mathrm{Kr}$, and $\mathrm{Rb}$ contain significant contributions from two processes. While there are uncertainties in these assignments, it is clear that abundances in this charge region represent a superposition of contributions from several processes (and therefore sites) of nucleosynthesis. In addition, the "Wolf-Rayet" model ${ }^{5}$ for cosmic ray origin, designed to explain anomalies in the elemental and isotopic composition of lighter nuclei ${ }^{6}$, also predicts a number of sizeable isotopic anomalies in these elements, including enhanced abundances of both isotopes of $\mathrm{Cu}$ and $\mathrm{Ga}$, and of ${ }^{67} \mathrm{Zn},{ }^{70} \mathrm{Ge},{ }^{82} \mathrm{Kr}$, and ${ }^{86} \mathrm{Sr}$. The charge region from $\mathrm{Z}=29$ to $\mathrm{Z}=40$ is relatively free of "secondary" production because there are no abundant heavier nuclei, and HEAO-3 studies ${ }^{7}$ indicate that "primaries" should account for $\sim 70 \%$ of the observed flux of nuclei with $31 \leq \mathrm{Z} \leq 40$. This charge region therefore presents an opportunity to isolate the contributions of several nucleosynthesis processes to cosmic ray source material.

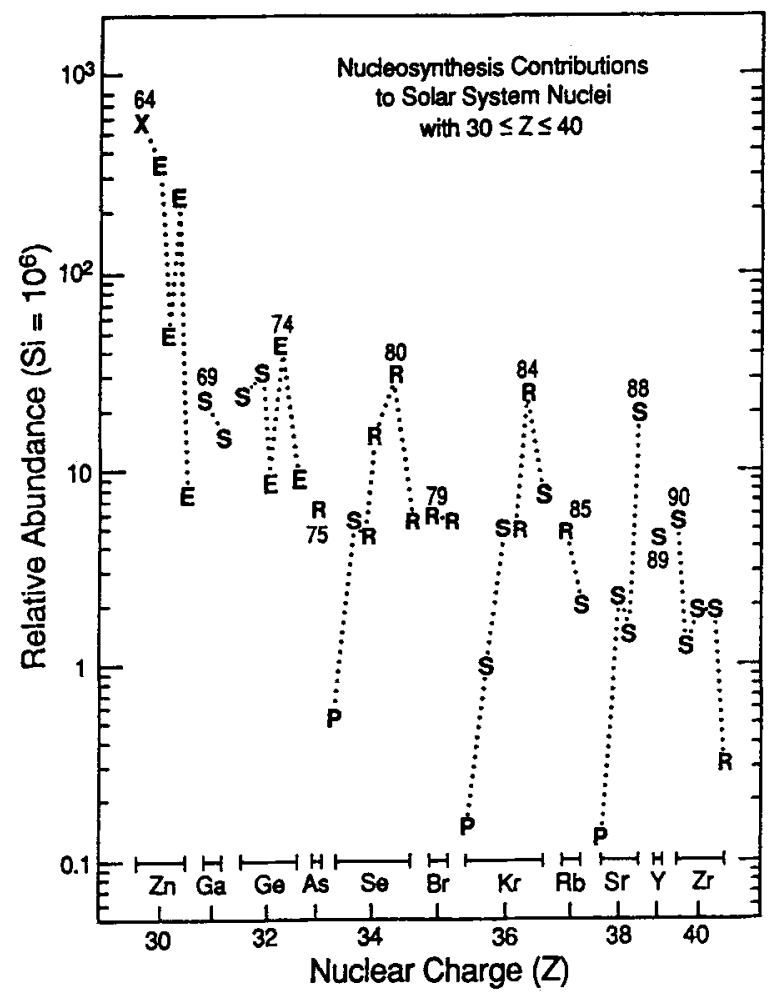

Figure 1: The relative abundances of the nuclides with $30 \leq Z \leq 40$ as compiled for "solar system" material ${ }^{4}$ are shown on a scale where $\mathrm{Si}=10^{6}$. Each isotope is labeled with a letter representing the process that produces the largest fraction of its solar system abundance, as tabulated by Anders and Grevesse ${ }^{4}[r, s$, e, and $p$ processes and explosive nucleosynthesis $(x)]$. The isotopes of a given element are connected by a dotted line with the mass number of the most abundant isotope labeled. 


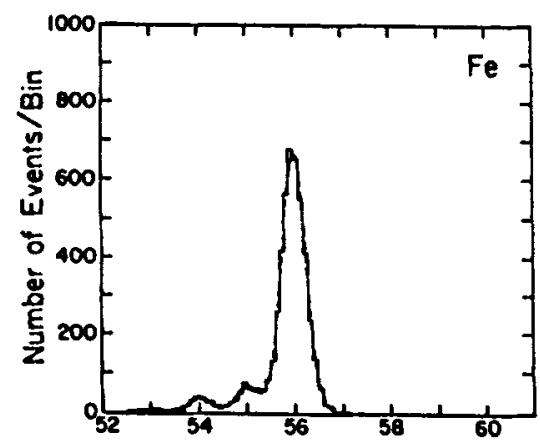

\section{Bevalac Calibration}

Caltech ISEE-3 Instrument

$\left(\sigma_{\mathrm{m}} \approx 0.23 \mathrm{amu}\right)$
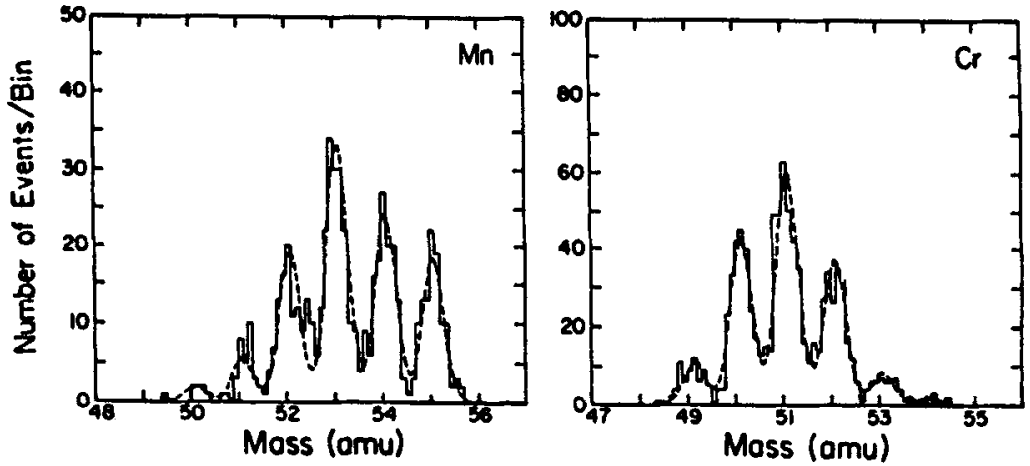

Figure 2: Mass distributions of $\mathrm{Cr}, \mathrm{Mn}$, and $\mathrm{Fe}$ isotopes obtained in a Bevalac calibration of the Caltech Heavy Isotope Spectrometer that was launched on ISEE-3. The observed mass resolution (dashed fit to data) is $\sim 0.23 \mathrm{amu}$.

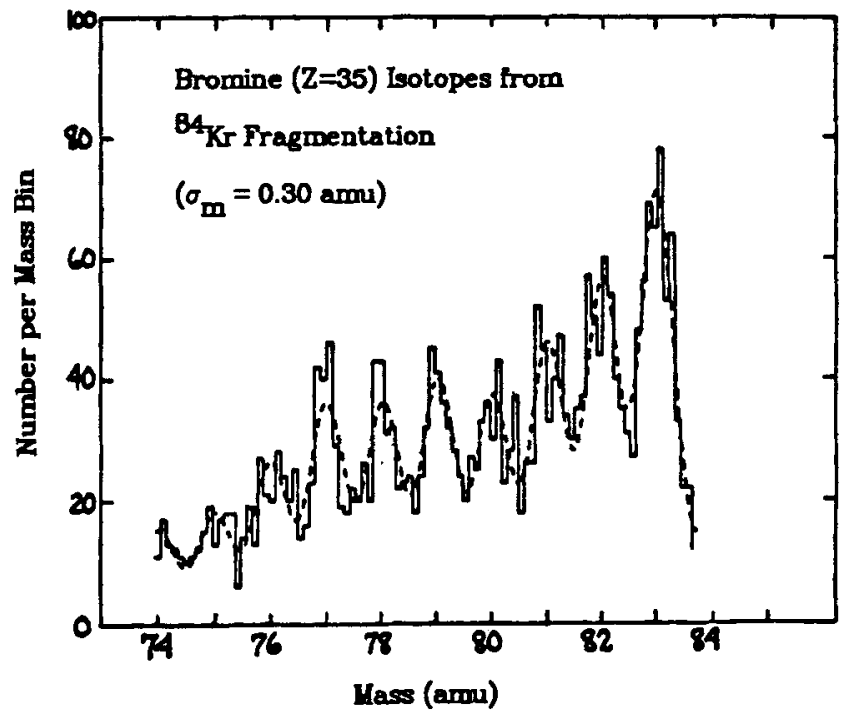

Figure 3: Mass histogram of $\mathrm{Br}$ isotopes obtained in a calibration of a large area solid state detector telescope at the Bevalac. The observed mass resolution of $0.30 \mathrm{amu}$ (dashed fit to data) is expected to be improved when corrections for detector nonuniformity are applied. 
Trans-Fe nuclei also present several new opportunities for studying cosmic ray propagation. There are pure electron capture nuclei with $31 \leq \mathrm{Z} \leq 40$ that might be used as probes of the density of the cosmic ray propagation region(s) ${ }^{8}$, and of possible energy-changing processes in the interplanetary and interstellar medium. In addition the element technicium ( $Z=43)$, which has no stable isotopes, has been suggested as a cosmic ray clock $^{9}$.

Isotope studies of UH nuclei require an instrument with both sufficient collecting power and good mass resolution. One possible approach is based on a combination of multi-detector arrays of large-area silicon solid state detectors combined in a module with a scintillating optical-fiber trajectory (SOFT) detector hodoscope, a concept that is under study for ACE. Silicon detectors have already demonstrated the capability to separate isotopes of nuclei up to $Z=36$ with an rms mass resolution of $\leq 0.3$ amu (see, e.g., Figures 2 and 3), while $200-\mu$ m thick SOFT detectors have demonstrated a position resolution of $\sim 70 \mu \mathrm{m}$ for $\mathrm{Fe}$ nuclei ${ }^{10}$. With a combination of these devices it should be possible to resolve isotopes at least through $Z=40$ over the approximate energy range from $\sim 100$ to $\sim 700 \mathrm{MeV} / \mathrm{nuc}$, and also abundant isotopes of heavier nuclei. The collecting power of such an instrument would depend on the number of modules, but a reasonable module size would yield $>10^{6} \mathrm{Fe} / \mathrm{yr}$, more than 10 times that of $\mathrm{ACE}$, and sufficient so that in a 3-year mission each module would give several thousand events each of $Z=29$ and $Z=30$, and $>1000$ nuclei total with $Z=31$ to 40 . A test of this concept. is planned for the Bevalac in 1990 .

Acknowledgements: This work was supported NASA grant NGR 05-002-160 at Caltech, and by NASA grants NGR 26-008-001 and NAG 5-846 and the McDonnell Center for Space Sciences at Washington University. We appreciate the assistance of B. W. Gauld and T. L. Garrard.

\section{REFERENCES}

1) Stone, E. C., et al., this volume (1989).

2) Price, P. B., this volume (1989).

3) Binns, W. R., T. L. Garrard, M. H. Israel, J. Klarmann, E. C. Stone, and C. J. Waddington, in Cosmic Abundances of Matter, Ed. C. J. Waddington, AIP Conference Proceedings \#183, New York, p. 147 (1989).

4) Anders, E., and N. Grevesse, Geochimica et Cosmochimica Acta 53, 197 (1989).

5) Prantzos, N., M Arnould, J. P. Arcoragi, and M. Casse, Proc. 19th Internat. Cosmic Ray Conf. (La Jolla), 3, 167 (1985).

6) Mewaldt, R. A., in Cosmic Abundances of Matter, Ed. C. J. Waddington, AIP Conference Proceedings \#183, New York, p. 124 (1989).

7) Garrard, T. L., private communication (1989).

8) Letaw, J. R., J. H. Adams, Jr., R. Silberberg, and C. H. Tsao, Astrophys. Sp. Sci. 114, 365 (1985).

9) Drach, J., and M. H. Salamon, Ap. J. 319, 237 (1987).

10) Davis, A. J., P. L. Hink, W. R. Binns, J. W. Epstein, J. J. Connell, M. H. Israel, J. Klarmann, and V. Vylet, Nuc. Instrum. Meth. A27 6, 347 (1989). 